\title{
Delusions and Three Myths of Irrational Belief
}

\section{Lisa Bortolotti}

Abstract This chapter addresses the contribution that the delusion literature has made to the philosophy of belief. Three conclusions will be drawn: (1) a belief does not need to be epistemically rational to be used in the interpretation of behaviour; (2) a belief does not need to be epistemically rational to have significant psychological or epistemic benefits; (3) beliefs exhibiting the features of epistemic irrationality exemplified by delusions are not infrequent, and they are not an exception in a largely rational belief system. What we learn from the delusion literature is that there are complex relationships between rationality and interpretation, rationality and success, and rationality and knowledge.

In the preparation of this chapter, Lisa Bortolotti acknowledges the support of project PERFECT, funded by a European Research Council Consolidator Award (grant agreement 616358). She is also grateful to Martin Davies and Matthew Broome for teaching her about delusions.

L. Bortolotti $(\bowtie)$

Philosophy Department and Institute for Mental Health, University of Birmingham, Birmingham, UK e-mail: L.Bortolotti@bham.ac.uk

(C) The Author(s) 2018

L. Bortolotti (ed.), Delusions in Context, https://doi.org/10.1007/978-3-319-97202-2_4 
Keywords Delusion $\bullet$ Belief $\bullet$ Rationality $\bullet$ Epistemic functionality $\bullet$ Psychological wellbeing $\bullet$ Mental health $\bullet$ Success $\bullet$ Sense of agency

\subsection{Lessons From DELUSIONS}

In recent years, the focus on delusions in the philosophical literature has contributed to dispel some myths about belief, making room for a more psychologically realistic picture of the mind. What do we know about delusions that can inform our understanding of how beliefs are adopted and maintained, and how they influence behaviour? What have philosophers learnt from delusions? An entire volume could be dedicated to this issue alone, but in this chapter I will focus on three core philosophical claims about epistemically irrational beliefs that our knowledge of delusions have successfully challenged.

First, our beliefs do not need to be epistemically rational to be used in the attempt to interpret our behaviour. The ascription of an epistemically irrational belief to us often contributes to the process of explaining and predicting what we do. Second, our beliefs do not need to be epistemically rational to have a positive impact on our psychological wellbeing or understanding. Sometimes, an epistemically irrational belief has some long- or short-term epistemic benefit because it shields us from anxiety or supports our sense of agency. Third, beliefs exhibiting the features of epistemic irrationality exemplified by delusions are not infrequent, and they are not an exception. Optimistically biased beliefs about ourselves, for instance, may also be poorly supported by the evidence available to us, and resistant to the evidence that becomes available to us at a later stage. Yet, they are very common and widely regarded as adaptive. This suggests that epistemic irrationality cannot account for the pathological nature of delusions.

In discussions about interdisciplinary projects involving philosophers, it is not uncommon to identify the role of the philosopher with the conceptual tidying up and clarifying that are often deemed to be necessary in complex empirical investigations, or with the capacity to place a timely investigation within a wider historical context. By all means, such roles are important and philosophers are well placed to assist. However, as others have observed (e.g., Fulford, Stanghellini, \& Broome, 2004), the role of the philosopher does not need to be so narrowly confined. Philosophers can also help develop a field in a certain direction, offering hypotheses to test and examining the wider implications of existing empirical results. 
In this chapter, I want to focus not on what philosophy can do for psychology and psychiatry, but on what psychology and psychiatry have done for philosophy. As I hope to show, there are opportunities for philosophers who engage in interdisciplinary projects to learn something from the empirical and clinical sciences about the nature of those phenomena that have traditionally been at the centre of philosophical investigation. A careful analysis of the results of focused empirical work on the relevant phenomena, and attention to detail in the relevant case studies can reveal the inadequacy of established philosophical theories and suggest new ways of looking at things.

Here I provide an example of this. I argue that the study of delusions has contributed to challenging some widely accepted assumptions in the philosophy of mind concerning the relationship between epistemic rationality and belief.

Although definitions of delusions vary to some extent, most definitions are based on the surface features of the delusions, and identify delusions as epistemically irrational beliefs. Let's see what it means for a delusion to be a belief that is epistemically irrational. First, what is a belief? Whereas our desire tells us how we would like things to be, our belief tells us how we take things to be. Thus, a belief is a mental state that purports to represent reality. If I believe that it rarely snows in London, I am committed to the truth of the claim that it rarely snows in London. Typically, our beliefs manifest in our verbal and non-verbal behaviour. For instance, I may decide that it is not a good idea to buy snow boots if I am going to spend most of the winter in London.

There are several distinct accounts of epistemic irrationality in the philosophical literature, but the central idea is that epistemic irrationality concerns the relationship between a belief and the evidence for it. ${ }^{.}$The notion of epistemic irrationality I am going to work with for the purposes of this chapter is as follows: we are epistemically irrational when (1) we do not have evidence supporting our beliefs prior to adopting them; or (2) we are not responsive to evidence against our beliefs that becomes available to us after their adoption.

\footnotetext{
${ }^{1}$ See for instance: "By epistemic rationality, I mean, roughly, the kind of rationality which one displays when one believes propositions that are strongly supported by one's evidence and refrains from believing propositions that are improbable given one's evidence" (Kelly, 2003, p. 612).
} 
According to some of the most influential definitions in the clinical and empirical literature, delusions are epistemically irrational beliefs. ${ }^{2}$ One good example of this general trend is the definition we find in the Diagnostic and Statistical Manual of Mental Disorders (DSM). The DSM-V says that delusions are "based on incorrect inference about external reality that are firmly sustained despite what almost everyone else believes and despite what constitutes incontrovertible and obvious proof or evidence to the contrary" (APA, 2013). The definition has been rightly criticised for the terminology it uses (Are all delusions based on inference? Can beliefs be proven to be false?) and for other good reasons (e.g., Coltheart, 2007, p. 1043), but it captures some aspects of the nature of delusions. In particular, both features of epistemic irrationality are present in the definition: delusions are ill-grounded and are not abandoned in the face of obvious proof or evidence against them.

If delusions are epistemically irrational beliefs, then they will share the characteristics typically attributed to epistemically irrational beliefs. But do they?

I will focus on three claims that are often regarded as safe assumptions about epistemically irrational beliefs in the philosophy of mind, and ask to what extent they are true of delusions. The first claim is that, due to our epistemically irrational beliefs, our behaviour is either impossible or difficult to understand, and other people's attempts to interpret or predict our actions on the basis of our beliefs are destined to fail. The second claim is that our epistemically irrational beliefs have negative consequences for our psychological and epistemic status, by compromising both our wellbeing and our access to the truth, and thus they should be challenged by default. The third claim is that epistemically irrational beliefs are an anomaly to be explained away, an exception in our largely rational belief system. Indeed, it is only because epistemic irrationality is not widespread that we can have intentional agency at all.

I will argue in this chapter that the three claims about epistemically irrational beliefs are not compatible with what we know about delusions. Moreover, they are inaccurate and misleading when applied to a number

\footnotetext{
${ }^{2}$ See for instance: "A person is deluded when they have come to hold a particular belief with a degree of firmness that is both utterly unwarranted by the evidence at hand, and that jeopardises their day-to-day functioning" (McKay et al., 2005, p. 315) and: "Delusions are generally accepted to be beliefs which (a) are held with great conviction; (b) defy rational counter-argument; and (c) would be dismissed as false or bizarre by members of the same socio-cultural group" (Gilleen \& David, 2005, pp. 5-6).
} 
of beliefs that are commonly regarded as epistemically irrational but are not delusional. In other words, they are either claims that must be qualified, or myths that must be left behind.

In Sects. 4.2 and 4.3, I explain how the study of delusions shows not only that epistemically irrational beliefs can be understood, but that it is often via the attribution of those beliefs to us that our behaviour can be explained and predicted. Even so-called 'bizarre delusions' can be understood in context and appealed to, both in the explanation of past behaviour and in the prediction of future behaviour. Thus, it is implausible to hold that epistemically irrational beliefs always or by necessity compromise interpretation.

In Sects. 4.4, 4.5 and 4.6, I offer some reasons to qualify the claim that irrational beliefs have negative consequences from a psychological and epistemic point of view. In some cases, irrational beliefs can have significant psychological and even epistemic benefits. In particular, delusional beliefs have some (obvious, long-term) costs and some (less obvious, shorter-term) benefits. Delusions appear not to be well-supported by evidence, are resistant to counterevidence and counterargument, and can seriously disrupt functioning. Depending on their content, they can be a source of anxiety and distress. But they can also make a contribution to our sense of competence and coherence, and, in the critical situation in which they often emerge, they can even support our engagement with the surrounding physical and social environment after uncertainty, trauma, or abuse. Thus, as counter-intuitive as it may sound, in those circumstances it may be unwise to challenge delusions.

Although philosophers accept that some false and irrational beliefs can be useful in some contexts, they are very resistant to the idea that false and irrational beliefs might have some positive role to play from an epistemic point of view. Surely, false and irrational beliefs take us further from the truth. How can they contribute to the achievement of epistemic goals, such as the acquisition, retention, and use of relevant information? I will show that some false and irrational beliefs can play a positive epistemic function. In particular, some delusions serve as an emergency response to a break-down of epistemic functionality.

In Sect. 4.7, I challenge the claim that irrational beliefs are the exception to the rule, a relatively rare occurrence in our largely rational belief systems. Although clinically significant delusions are not widespread, beliefs sharing the same epistemic features as delusions, and thus falling short of standards of epistemic rationality, are common. Here I will refer 
to positive illusions (Taylor, 1989) generating overly optimistic beliefs about ourselves that are not well-supported by evidence, are resistant to counterevidence, and often misrepresent reality. What distinguishes delusions from optimistically biased beliefs and other epistemically irrational beliefs is an interesting question that has not been satisfactorily answered yet. But the discussion in this chapter will give us reason to believe that the source of the alleged pathological nature of delusions cannot be their epistemic irrationality.

In Sect. 4.8, I will examine the implications of dispelling the three myths of epistemically irrational belief discussed in the previous sections. How does the rejection of the assumptions surrounding epistemically irrational beliefs affect what we know about the mind and how we view mental health?

\section{2 “Irrational Beliefs Make Interpretation IMPOSSIBLE"}

If a man says that there is a full-scale nuclear reactor inside himself, or if a woman says that she is always pregnant and giving birth to a series of Messiahs, you may wonder whether they really mean what they say. ${ }^{3}$ That is because what these people say sounds not just implausible, but impossible.

On an influential view in philosophy of mind, rationality and understanding go hand in hand. More precisely, our behaviour can be interpreted, explained, and predicted in intentional terms, that is, by reference to our beliefs, desires, intentions, and so on, only if it meets some basic standards of rationality. The view is reflected in two influential approaches to interpretation, the so-called principle of charity (Davidson, 1980) and the intentional stance (Dennett, 1987).

If we are intelligibly to attribute attitudes and beliefs, or usefully to describe motions as behavior, then we are committed to finding, in the pattern of behavior, belief, and desire, a large degree of rationality and consistency. (Davidson, 1980, p. 237)

When we are not [rational], the cases defy description in ordinary terms of belief and desire. (Dennett, 1987, p. 87)

${ }^{3}$ These examples are discussed in Bortolotti and Broome (2012). 
Although for Davidson and Dennett the demands of epistemic rationality do not exhaust the rationality constraints on belief attribution, epistemic rationality plays a central role in the development of their views on belief and intentionality.

There are several versions of the idea that rationality and interpretation go together, some stronger (according to which it is impossible to interpret irrational behaviour) and other weaker (according to which it is especially difficult to interpret irrational behaviour). Here is one version of the idea that there is a rationality constraint on the ascription of beliefs:

Propositional attitudes have their proper home in explanations of a special sort: explanations in which things are made intelligible by being revealed to be, or to approximate to being, as they rationally ought to be. (McDowell, 1985 , p. 389)

\subsection{Bizarre Delusions}

So-called bizarre delusions seem to be the perfect illustration of the claim that irrationality compromises interpretation and understanding. In the DSM-V, delusions are described as bizarre "if they are clearly implausible and not understandable to same-culture peers and do not derive from ordinary life experiences" (APA, 2013). 'Bizarre' delusions are epistemically irrational in that they are neither well-supported by the existing evidence, nor responsive to new evidence; moreover, they are implausible, typically conflicting with other things we are committed to. ${ }^{4}$

Karl Jaspers (1963) describes some delusions (those delusions that involve a radical transformation of both experience and meaning) as $\mathrm{un}^{-}$ understandable. The definition of delusions in the DSM-V (APA, 2013, p. 87) reflects this approach: bizarre delusions are an instance of irrational behaviour ("fixed beliefs that are not amenable to change in light of conflicting evidence") that cannot be interpreted ("clearly implausible and not understandable to same-culture peers").

But the view that our behaviour cannot be interpreted in intentional terms unless the belief we seem to express is rational has unattractive

\footnotetext{
${ }^{4}$ See for instance: "Rationality is a normative constraint of consistency and coherence on the formation of a set of beliefs and thus is prima facie violated in two ways by the delusional subject. First she accepts a belief that is incoherent with the rest of her beliefs, and secondly she refuses to modify that belief in the face of fairly conclusive counterevidence and a set of background beliefs that contradict the delusional belief" (Gerrans, 2000, p. 114).
} 
consequences. If we cannot be interpreted as believing what we say, and our behaviour cannot be explained or predicted on the basis of our reports, when they are taken literally, then what should our interpreters do?

They could attempt to rationalise what we say, that is, assume that we do not mean what we literally say. As a result, they could make an alternative attribution to us that no longer violates rationality constraints. They could make sense of the man reporting that he has a nuclear reactor inside himself by 'correcting' his report: "Maybe, what he means is that he feels like he has a reactor inside himself because he feels that there is something wrong with him." The same strategy of rationalisation could be adopted in the case of the woman reporting that she has given birth to an endless series of Messiahs. "Maybe, what she means is that she is willing to give birth to an endless series of Messiahs if God asks her to, because she want to do God's will."

In cases of apparent irrationality, philosophers committed to there being a rationality constraint on belief ascription suggest that interpreters need to find a way to rationalise the report, to 'correct the mistake' ${ }^{5}$ The problem is that the rest of the behaviour of people with 'bizarre' delusions often makes sense only if their reports are taken at face value, literally, and not as metaphorical expressions of discomfort or desire. It is because the content of the delusion is believed, often with conviction, that it has such a significant, and often disruptive, impact on their lives.

The man who reported that he had a nuclear reactor inside himself was concerned about the presence of the nuclear reactor, and experienced frustration when others did not believe him. The man would not have been as concerned if the presence of a reactor inside himself had just been a metaphor, and he would not have felt frustration at other people's disbelief. The woman who reported that she had given birth to an endless series of Messiahs felt privileged about her condition (Bortolotti \& Broome, 2012, p. 190). The woman would not have felt this way if she had just desired to give birth to Messiahs. It is because she believed that she had given birth to them that she felt privileged.

The strong feelings and persistent thoughts related to our 'bizarre' delusions are easier to understand in the context of our believing (as opposed

\footnotetext{
${ }^{5}$ See for instance: " $[. .$.$] when a mistake is agreed to have been made we will often look for,$ and find, a reason why it was made, not just in the sense of cause or regularity in its making but in the sense of some excuse which reconciles the mistake with the idea that, even in making it, the perpetrator was exercising his or her rationality" (Heal, 1998, p. 99).
} 
to imagining or desiring) that we are in a very peculiar situation. And the content of the delusional beliefs may not appear as bizarre in the context of our experiences prior to the adoption of the delusional beliefs. In many circumstances delusions make sense. For instance, those who develop delusions of persecution often have been abused or mistreated in their past and so their tendency to see other people as hostile can be easily explained in context (see Gunn \& Bortolotti, 2018).

Some background knowledge about our significant life events combined with attention to how we talk about our experiences can help the interpreter make sense of 'bizarre' delusions. This idea can be extended to a wider range of unusual beliefs. For instance, it is easier to understand why we claim to have been abducted by aliens if interpreters take into account our experiences and cultural background (see Bortolotti, Gunn, \& Sullivan-Bissett, 2016). Experiences of 'abduction' can be caused by a phenomenon called 'awareness during sleep paralysis' (ASP) and by hypnopompic hallucinations. During Rapid Eye Movement (REM) sleep, we are immobilized and can wake up before the paralysis has disappeared, realising that we are unable to move. Sleep paralysis can be accompanied by hypnopompic hallucinations, which means that we are also hallucinating sights and sounds. Faced with these experiences, we search for an explanation, and we may be inclined to believe that we have been abducted by aliens if we belong to a sub-culture where the idea of intelligent aliens coming in contact with humans is not ruled out.

\section{4 "Irrational Beliefs Are Always Bad for Us"}

Delusional beliefs are a common example of irrationality but are also a perfect illustration of beliefs that can be harmful, generating distress, and severely disrupting our lives. Many of the people who come to the attention of healthcare professionals and are diagnosed with delusions do not sleep properly, experience social withdrawal, cannot keep their jobs or continue their studies, and cause concern to their families, employers, neighbours, sometimes even the police.

Although there are cases where some people feel at least temporarily empowered by or privileged about the content of their delusions (as the woman who thought she had given birth to an endless series of Messiahs), for the great majority of people delusions are a source of unhappiness. Thus, it is natural to link the epistemic irrationality of the delusions to their harmfulness. Isn't it because they are so divorced from reality that 
delusions end up affecting our lives so negatively? I hope to show here that the link between harmfulness and epistemic irrationality is tempting, but is ultimately an oversimplification.

I am going to offer some examples of delusions that are harmful, in that they generate clashes with reality that are a source of anxiety and distress, but also play a protective or defensive role, temporarily ameliorating a critical situation. Let's start with the most obvious example, so-called motivated delusions (Bortolotti, 2015). How the delusions are described gives it away: these are beliefs that we may be motivated to hold on to, because they represent not the reality we find ourselves in, but a reality we would prefer to be in.

\subsection{Motivated Delusions}

An example of a delusion with a defensive function is the case of Reverse Othello syndrome detailed in P.V. Butler and further discussed by Ryan McKay, Robyn Langdon, and Max Coltheart. BX was a talented musician who became quadriplegic after a tragic accident. He believed that he was still in a satisfying relationship, when in fact the woman who had been his partner had left him and started a relationship with someone else (McKay, Langdon, \& Coltheart, 2005). BX's belief in the fidelity of his former partner and the continued success of his relationship was very resistant to counterevidence. BX believed that his relationship was going well, even though his former partner refused to communicate with him (Butler, 2000, p. 86).

The Reverse Othello syndrome can be seen as a special case of erotomania. In erotomania, we come to believe that another person, often of a perceived higher status, is in love with us when there is no clear evidence in support of that belief. Here is a more typical case of erotomania. LT was a young woman who became obsessed with the idea that a fellow student was in love with her although the two had never spoken to each other. She explained that the student would send her love messages and marriage proposals via the TV, the colours of dresses, and car licence plates (Jordan \& Howe, 1980). Although the young man was asked to talk to LT on the phone, clarifying that he had no intention to marry her, LT remained convinced that he loved her, and came to believe that the man on the phone was another person.

In the cases of $\mathrm{BX}$ and $\mathrm{LT}$, what are their delusions protecting them from? It is not easy to say, but one can speculate from the further details 
provided in the case studies. Butler argues that for BX the appearance of the delusion "may mark an adaptive attempt to regain intrapsychic coherence and to confer meaning on otherwise catastrophic loss or emptiness" (Butler, 2000, p. 90). BX did feel a sense of loss after the accident because he acquired a severe disability compromising his future aspirations. The belief that his relationship had not ended was both false and resistant to counterevidence, but gave him hope and strength at a critical time.

LT's mother described her as quiet and lonely, and explained that one of her brief relationships had ended just before she started believing that her fellow student was in love with her. The break up had caused her significant distress (Jordan \& Howe, 1980, pp. 982-3). The belief that she was loved and desired might have protected LT from low mood, following a long history of low self-esteem and a recent, painful rejection.

The lesson from motivated delusions is that an epistemically irrational belief can prevent people who have experienced traumatic or emotionally distressing events from becoming depressed. As the belief enhances reality, in the short run it may be a psychologically adaptive response. By enabling us to continue to interact with the surrounding environment (albeit imperfectly), motivated delusions can also support our epistemic functionality, that is, our capacity to acquire, retain, and use relevant information. Along these lines, Butler and his team speculated that BX's Reverse Othello syndrome supported his motivation to engage in rehabilitation, and noted that the delusion faded away soon after the rehabilitation programme had ended.

The adoption of motivated delusions seems to provide some temporary relief from low mood and anxiety and thus protecting from lack of concentration, irritability, social isolation, and emotional disturbances. One might think that the case of motivated delusions is especially well suited to the purpose of showing that some epistemically irrational beliefs can be good for us. But it has been suggested that other types of delusions can also play an adaptive role, at least in the short term.

\subsection{Delusions in Schizophrenia}

Consider the following example, adapted from the one originally presented by Schneider in Clinical Psychopathology (1959). You are taking a walk in your hometown when you notice a dog on the steps of a Catholic church. While you pass the front of the church, the dog gets up on his hind legs. Then he moves his front paw forward. What do you make of 
this? Probably nothing. But what if you felt that the dog's action was meant for you? Maybe you would start thinking about other events involving churches that you witnessed recently. Maybe the previous encounters were also meant for you and led up to today's event. Maybe the dog was trying to communicate something. Maybe he was delivering a message from God, revealing that you were chosen to carry out an important mission.

The hypothesis that the dog is a messenger from God is far-fetched, you can grant that. If one of your friends had come up with such a story you would not believe her, and so it is not a surprise for you if your friends do not believe you when you tell them. But you just know it is true. It explains everything. Knowing why the dog behaved as he did dissolves at last the anxiety and uncertainty that had become a constant feature of your everyday experiences: feeling that something important was about to happen, and the dread of not knowing what it was. When you realize that the dog is delivering a message from God, you feel relieved and empowered. God has found a secret and effective way to communicate with you.

Classical authors such as Karl Jaspers (Jaspers, 1963) and Klaus Conrad (Mishara, 2010), and contemporary authors such as Glen Roberts (Roberts, 1991, 2006), and Aaron Mishara and Phil Corlett (Mishara \& Corlett, 2009), have argued that elaborated delusions in schizophrenia can be seen as either an emergency response or a revelation, putting an end to a situation of great uncertainty and anxiety. To start with, the adoption of a delusional hypothesis may support epistemic functionality by fostering a new attitude towards experience. We feel that it is in our power to understand what is going on in our lives. An interesting study (Bergstein, Weizman, \& Solomon, 2008) has shown that people with elaborated delusions in the acute stage of psychosis have a strong sense of coherence, a psychological construct encompassing intellectual curiosity and a sense of self-efficacy and purpose. ${ }^{6}$ Arguably, such an attitude towards experience is more conducive to the acquisition and exchange of information than the state of passive, anxious uncertainty that characterizes the prodromal phase of psychosis.

\footnotetext{
${ }^{6}$ The sense of coherence is defined as "a global orientation that expresses the extent to which one has a pervasive, enduring though dynamic, feeling of confidence that (1) the stimuli deriving from one's internal and external environments are structured, predictable, and explicable; (2) the resources are available to one to meet the demands posed by these stimuli; and (3) these demands are challenges, worthy of investment and engagement" (Antonovsky, 1987, p. 91).
} 
Then, the adoption of the delusion may have positive effects on the habitual and automated processes that are conducive to learning. One common view about the adoption of delusional beliefs is that, in the prodromal stage of psychosis, we experience random events as especially important to us and cannot understand why. ${ }^{7}$ Such experiences can become distressing and demand an explanation (such as, "God is using the dog to send me a message"). The delusion can be that explanation, enabling us to overcome uncertainty and making sense of what goes on around us. ${ }^{8}$

Thus, it has been argued that some delusions are adaptive not only psychologically, by temporarily reducing anxiety, but also in other ways (Mishara \& Corlett, 2009). The suggestion is that delusions allow us to keep in touch with our environment. This claim can be explained by reference to how delusions are adopted. In one influential hypothesis about delusion formation, during the prodromal stage of psychosis, prediction-error signals are produced when there is no real mismatch between prediction and actual inputs. We feel that something significant is happening because our experience does not match our predictions. When this happens, our internal model of the world is thought to be incorrect and undergoes revision. As a result of excessive prediction-error signals, automated and habitual learning is compromised and conscious and controlled processes take over instead.

When the delusion is formed, not only does it put an end to overwhelming anxiety, but it also helps overcome the sense of unpredictability caused by the inaccurate coding of prediction errors. What was experienced as salient is no longer seen as requiring attention, because the delusion can explain it. Thus, the processes underlying automated and habitual learning can resume. This also makes sense of the persistence of delusions. The belief is reinforced every time a new prediction error is registered, given that it has become the default explanation of the unexpected data.

\footnotetext{
${ }^{7}$ See for instance: "This general delusional atmosphere with all its vagueness of content must be unbearable. Patients obviously suffer terribly under it and to reach some definite idea at last is like being relieved of some enormous burden [...] The achievement brings strength and comfort [...] No dread is worse than that of danger unknown" (Jaspers, 1963, p. 98).

${ }^{8}$ See for instance: "Delusion formation can be seen as an adaptive process of attributing meaning to experience through which order and security are gained, the novel experience is incorporated within the patient's conceptual framework, and the occult potential of its unknownness is defused" (Roberts, 1992, p. 305).
} 
The delusions [...] involve a 'reorganization' of the patient's experience to maintain behavioral interaction with the environment despite the underlying disruption to perceptual binding processes [...] At the Aha-moment, the 'shear pin' breaks, or as Conrad puts it, the patient is unable to shift 'reference-frame' to consider the experience from another perspective. The delusion disables flexible, controlled conscious processing from continuing to monitor the mounting distress of the wanton prediction error during delusional mood and thus deters cascading toxicity. At the same time, automatic habitual responses are preserved, possibly even enhanced. (Mishara \& Corlett, 2009, p. 531)

We saw in this section that delusions can play an adaptive role, by offering psychological relief from negative emotions and by restoring an epistemically beneficial engagement with reality (Bortolotti, 2016) after the severe disruption due to hypersalience. The consideration of these positive effects prompts us to challenge the claim that epistemically irrational beliefs cannot be good for us.

\section{7 "Irrational Beliefs Are the Exception"}

We saw that delusions are paradigmatic examples of epistemically irrational beliefs. But not all epistemically rational beliefs are unusual or infrequent. Although the type of delusional belief that attracts the attention of healthcare professionals is relatively rare, beliefs that satisfy the conditions for epistemic rationality and share significant epistemic features with delusions can be easily found in the non-clinical population. The prejudiced belief that members of a certain ethnic group are violent or lazy is not obviously less epistemically irrational than the delusional belief that our neighbor is a spy paid by the government to follow our movements. Superstitious beliefs about nights of full moon causing accidents share many of the epistemic features of delusions: they are badly supported by the available evidence, and they are resistant to counterevidence and counterargument. The reason why prejudiced and superstitious beliefs come across as less puzzling than delusions is that they very widespread and not particularly distressing to those who report them. ${ }^{9}$ Here I am going to focus on the literature suggesting that we have a tendency to adopt self-enhancing

\footnotetext{
${ }^{9}$ See Bortolotti (2009, chapter 3 ) for a more comprehensive discussion of non-delusional beliefs that are epistemically irrational.
} 
beliefs and make overly optimistic predictions about our future (Jefferson, Bortolotti, \& Kuzmanovic, 2017).

Different types of 'positive illusions' are discussed in psychology (Taylor, 1989). We experience the illusion of control when we overestimate our capacity to control independent, external events. We think that we have a better chance at winning when we roll the dice in a betting situation. We experience the better-than-average effect or have the illusion of superiority when we regard ourselves as above average and overrate our performance relative to others in a variety of domains. For instance, many believe that they are above average drivers. The optimism bias is a tendency to predict that our future will be largely positive and will yield progress, and that negative events will not be part of our lives. We all underestimate the likelihood of experiencing divorce or developing a serious health condition during our lives.

Are optimistically biased beliefs an instance of irrationality? Here is a reminder that epistemically irrational beliefs can be either true or false, but what makes them irrational is that they are not well supported by the evidence available to us or are insufficiently responsive to new evidence after being adopted. Unrealistically optimistic beliefs fit this description. Often the evidence on which we base our self-related judgements is biased, in that we tend to remember our past successes and forget our failures, or we tend to interpret negative feedback in a positive light. Moreover, we asymmetrically update beliefs about ourselves, taking into account evidence for desirable outcomes but ignoring evidence for undesirable outcomes (Sharot, Korn, \& Dolan, 2011). There is an element of motivationally driven distortion of the evidence in the way unrealistically optimistic beliefs are adopted.

To some extent, optimistically biased beliefs can be modified. It has been shown that there are interventions that are at least partially successful in reducing or controlling the extent to which self-related beliefs and predictions are optimistic. For instance, via introspective reflection we can control self-enhancing beliefs at least in the short-term, and self-assessment and predictions about our future are more accurate when we are held accountable for our judgements (Sedikides, Horton, \& Gregg, 2007).

Shelley Taylor makes the explicit claim that optimistically biased beliefs are not as fixed as delusional beliefs are, but are flexible and can be adjusted (Taylor, 1989). As we saw, we are known to update our self-related predictions in the light of new evidence, but we tend to do so to a greater extent when the new information is desirable and indicates that our previous 
estimates were pessimistic. Moreover, we tend to be more optimistic about events that we know we can partially control, and less optimistic just before receiving outcome feedback (Sweeny, Carroll, \& Shepperd, 2006). While we may give up our optimistic predictions in order to brace for bad news, we also tend to avoid situations that would cause disappointment, that is, situations in which our optimistic beliefs and predictions could be easily disproved (Armor \& Taylor, 1998; Neff \& Geers, 2013). Although this is evidence of some flexibility in optimistically biased beliefs, it does not support the claim that we are responsive to evidence in an epistemically rational way when it comes to self-related beliefs and predictions. Rather, the hypothesis is that optimism is strategically enhanced when fewer opportunities for it to be disconfirmed are available.

\subsection{Conclusion}

Here is what we have learnt from delusions: (1) it is not safe to assume that epistemically irrational beliefs compromise interpretation as it is possible for others to make sense of our behaviour by attributing delusional beliefs to us; $(2)$ it is true that epistemically irrational beliefs can be harmful, and delusions often are very harmful and disruptive, but we should also be open to the possibility that they may have some benefits from a psychological and epistemic point of view, and this might mean that to challenge them is not always the best course of action; (3) epistemically irrational beliefs are not rare and there are many beliefs that share the same epistemic features of delusions but are widespread in the non-clinical population.

Why does all of this matter? Suppose that the attribution of epistemically irrational beliefs contributes to our attempts to explain and predict each other's behaviour, and adopting some of these epistemically irrational beliefs is instrumental to our maintaining some engagement with the surrounding environment in critical situations, irrespective of whether the beliefs are unusual or mundane. Then, there are wide-ranging implications for philosophy, but also for our conception of delusions as symptoms of a mental health issue.

First, as philosophers who intend to gain a better understanding of the workings of the mind, we should be ready to reassess the nature of the links that we identify between core concepts. What is the relationship between rationality and interpretation, rationality and happiness, rationality and knowledge, rationality and success? Such questions have no straight-forward or general answer. We may find it intuitive and coherent 
with other things that we believe that irrationality leads to the paralysis of interpretation, ignorance, and misery, and in some cases that will happen. But if that is not always the case, then we should get to work and identify the more complex relationships there may be between having beliefs that are ill-grounded and impervious to counterevidence, and the goals we pursue in our lives. In what circumstances is an irrational belief important to us, enabling us to engage successfully with our environment? In what circumstances is it so harmful and disruptive that we should attempt to get rid of it as soon as we can?

Second, it is not obvious that epistemically irrational beliefs should be corrected, challenged, or regarded as a glitch in an otherwise rational belief system. The whole attitude towards such beliefs should change. We all have many epistemically irrational beliefs, and they are not always a sign that we lack credibility or we are mentally unwell. Rather, they are predictable features of human cognition (Puddifoot and Bortolotti, 2018). We are not unbiased in the way we weigh up evidence and we tend to be conservative once we have adopted a belief, making it hard for new contrary evidence to unsettle our existing convictions. Some delusions are just a vivid illustration of a general tendency that is widely shared and hard to counteract. Delusions, just like more common epistemically irrational beliefs, may be a significant obstacle to the achievements of our goals and may cause a rift between our way of seeing the world and other people's way. That is why it is important to develop a critical attitude towards their content. But it would be reckless not to acknowledge that there are situations in which such beliefs have a role to play, maybe allowing us to manage some very strong emotions or to respond to some adverse events that have a dramatic impact on our lives. In such situations, dismissing the belief as a mark of madness may not be the best course of action; at least, not before there is some understanding of how the belief emerged, and what role it has in our mental economy.

The picture I have sketched is a picture of continuity between so-called normal and abnormal cognition. Irrationality is a feature of normal cognition, and as such it cannot be the criterion of demarcation between beliefs that are 'healthy' and beliefs that are 'pathological'. In recent years, there have been many attempts to explain the pathological nature of delusions, and the general direction of such attempts has been to identify the problem with something other than the epistemic features of the delusional belief. For instance, Miyazono (2015) defends the view that delusions are pathological because they are harmful dysfunctions, that is, they 
are beliefs that negatively affect wellbeing and are caused by mechanisms that do not work properly. According to another account, by Petrolini (2017), the pathological nature of delusions is due to the person losing the capacity for relevance detection, that is, the capacity to determine which aspects of the environment are important within a given context.

These are interesting proposals which elucidate some aspects of delusions and deserve further consideration. But here is a more radical suggestion, maybe anticipating a new potential contribution that delusion research could make to the philosophy of the mind. What if there are no pathological beliefs? What if the locus of any pathology is the person as a whole? When the person is unwell, then there is a pathological state that needs to be addressed in order to restore health. The pathological state can manifest with unusual beliefs, hallucinations, emotional dysregulation, and so on, but such beliefs, experiences, and mood changes are not themselves pathological, as in other circumstances they may not cause any harm. It is in the context of what happens to the person as a whole that their role as symptoms can be assessed, and even when they contribute to the pathological state, they may have other roles to play that are either neutral or even positive in some respects.

\section{REFERENCES}

American Psychiatric Association. (2013). Diagnostic and statistical manual of mental disorders (5th ed.). Washington, DC: American Psychiatric Association.

Bergstein, M., Weizman, A., \& Solomon, Z. (2008). Sense of coherence among delusional patients: Prediction of remission and risk of relapse. Comprehensive Psychiatry, 49, 288-296.

Bortolotti, L. (2015). The epistemic innocence of motivated delusions. Consciousness and Cognition, 33, 490-499.

Bortolotti, L. (2016). The epistemic benefits of elaborated and systematised delusions in schizophrenia. British Journal for the Philosophy of Science, 67(3), 879-900.

Bortolotti, L., \& Broome, M. (2012). Affective dimensions of the phenomenon of double bookkeeping in delusions. Emotion Review, 4(2), 187-191.

Bortolotti, L., Gunn, R., \& Sullivan-Bissett, E. (2016). What makes a belief delusional? In I. Mac Carthy, K. Sellevold, \& O. Smith (Eds.), Cognitive confusions: Dreams, delusions and illusions in early modern culture. Cambridge, UK: Legenda.

Fulford, K. W., Stanghellini, G., \& Broome, M. (2004). What can philosophy do for psychiatry? World Psychiatry, 3(3), 130-135. 
Gerrans, P. (2000). Refining the explanation of the Cotard's delusion. In M. Coltheart \& M. Davies (Eds.), Pathologies of belief (pp. 111-122). Oxford, UK: Blackwell.

Gilleen, J., \& David, A. S. (2005). The cognitive neuropsychiatry of delusions: From psychopathology to neuropsychology and back again. Psychological Medicine, 35, 5-12.

Gunn, R., \& Bortolotti, L. (2018). Can delusions play a protective role? Phenomenology and the Cognitive Sciences, 17(4), 813-833.

Heal, J. (1998). Understanding other minds from the inside. In A. O'Hear (Ed.), Current issues in philosophy of mind. Cambridge, MA: Cambridge University Press.

Jaspers, K. (1963). General Psychopathology (7th ed., J. Hoenig \& M. W. Hamilton, Trans.). Manchester: Manchester University Press.

Jefferson, A., Bortolotti, L., \& Kuzmanovic, B. (2017). What is unrealistic optimism? Consciousness and Cognition, 50, 3-11.

Kelly, T. (2003). Epistemic rationality as instrumental rationality: A critique. Philosophy and Phenomenological Research, 66, 612-640.

McKay, R., Langdon, R., \& Coltheart, M. (2005). "Sleights of mind": Delusions, defences, and self-deception. Cognitive Neuropsychiatry, 10, 305-326.

Mishara, A. L. (2010). Klaus Conrad (1905-1961): Delusional mood, psychosis, and beginning schizophrenia. Schizophrenia Bulletin, 36, 9-13.

Mishara, A. L., \& Corlett, P. (2009). Are delusions biologically adaptive? Salvaging the doxastic shear pin. Behavioral and Brain Sciences, 32, 530-531.

Miyazono, K. (2015). Delusions as harmful malfunctioning beliefs. Consciousness and Cognition, 33, 561-573.

Petrolini, V. (2017). What makes delusions pathological? Philosophical Psychology, $30(4), 502-523$.

Puddifoot, K., \& Bortolotti, L. (2018). Epistemic innocence and the production of false memory beliefs. Philosophical Studies, 1-26.

Roberts, G. (1991). Delusional belief systems and meaning in life: A preferred reality? The British Journal of Psychiatry, 159, S19-S28.

Roberts, G. (1992). The origins of delusion. The British Journal of Psychiatry, 161, 298-308.

Roberts, G. (2006). Understanding madness. In G. Roberts, S. Davenport, F. Holloway, \& T. Tattan (Eds.), Enabling recovery: The principles and practice of rehabilitation psychiatry (pp. 93-111). London: Gaskell.

Sharot, T., Korn, C. W., \& Dolan, R. J. (2011). How unrealistic optimism is maintained in the face of reality. Nature Neuroscience, 14, 1475-1479.

Taylor, S. E. (1989). Positive illusions: Creative self-deception and the healthy mind. New York: Basic Books. 
Open Access This chapter is licensed under the terms of the Creative Commons Attribution 4.0 International License (http://creativecommons.org/licenses/ by $/ 4.0 /)$, which permits use, sharing, adaptation, distribution and reproduction in any medium or format, as long as you give appropriate credit to the original author(s) and the source, provide a link to the Creative Commons license and indicate if changes were made.

The images or other third party material in this chapter are included in the chapter's Creative Commons license, unless indicated otherwise in a credit line to the material. If material is not included in the chapter's Creative Commons license and your intended use is not permitted by statutory regulation or exceeds the permitted use, you will need to obtain permission directly from the copyright holder.

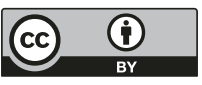

